\title{
Augmented Lagrangian method for imposing nonlinear multi freedom constraints in static analysis for frames using FEM
}

\author{
Vu Thi Bich Quyen*, Dao Ngoc Tien, Tran Thi Thuy Van, and Pham Van Dat \\ Faculty of Civil Engineering, Hanoi Architectural University, Km 10, Nguyentrai, Hanoi, Vietnam
}

\begin{abstract}
This paper focuses on the treatment of nonlinear multi freedom constraints using an augmented Lagrangian method in finite element analysis of frames. The process of imposing boundary constraints is developed by changing the assembly stiffness equation to produce a modified system of equation considering nonlinear multi freedom constraints. For imposing the nonlinear constraints two better methods are the penalty augmentation method and Lagrange multiplier adjunction method. But there are not free of disadvantages. Using penalty method has a disadvantage in the choice appropriate weight values that balance solution accuracy with the violation of constraint conditions. Using the Lagrange multiplier adjunction method requires additional unknowns, and more complicated storage allocation procedures. This research proposes the connection between these methods using the augmented Lagrangian method for imposing the nonlinear multi freedom constraints in finite element analysis of frame. Based on the Newton Raphson method the incremental-iterative algorithm for solving the nonlinear balanced equations is established.
\end{abstract}

\section{Introduction}

The establishment of solving algorithm for finite element analysis of frames is highly dependent on the implementation of the boundary constraints. Implementing boundary constraints is developed by applying constraints and changing the unmodified master stiffness equation to produce a modified system of equations based on the master stiffness equation $[1,2]$. The difficulty in constructing and solving the modified system of equations considerably increases in finite element analysis of systems having nonlinear multi freedom constraints. The better techniques suited to the implementation of nonlinear constraints are the penalty augmentation method and Lagrange multiplier adjunction method, employed in the solution of equality constrained optimization problems [3]. The penalty function method has the main advantage in its straightforward implementation and easily employed in the treatment of nonlinear constraints [4]. However, the penalty method has a disadvantage in the difficulty of selecting the weight values that balance solution accuracy with the violation of constraint conditions. In contrast to the penalty function method, the

* Corresponding author: bquyen1312@gmail.com 
Lagrange multiplier adjunction has the advantage of being exact, providing the constraints forces, not requiring guesses as regards weights and it can be effectively employed for treating nonlinear constraints [6]. But this method is not free of disadvantage. Using the Lagrange multiplier adjunction method requires additional unknowns, and more complicated storage allocation procedures. This research proposes using the augmented Lagrangian method $[7,8]$, the useful connection between penalty function and Lagrange multiplier methods, for imposing the nonlinear multi freedom constraints in finite element analysis of frame. Based on the augmented Lagrangian method, the increment equation is constructed for finite element analysis of frame having nonlinear multi freedom constraints. For solving the nonlinear modified system of equations, the incremental-iterative algorithm is established using the Newton Raphson techniques. Based on the proposed algorithm, the calculation program is written for numerical investigations.

\section{Problem formulation}

\subsection{Imposing nonlinear multi freedom constraints}

For imposing the nonlinear multi freedom constraint into the master stiffness matrix, consider the $\mathbf{n}^{\text {th }}$ degree of freedom system, designating:

- the global nodal displacement vector of frame system $\mathbf{u}=\left\{\mathrm{u}_{1}, \mathrm{u}_{2}, \ldots, \mathrm{u}_{n}\right\}^{T}, \mathbf{u} \in R^{n}$;

- the augmented potential energy of the unconstrained finite element model $\Pi(\mathbf{u})$

The nonlinear constrains equation of finite element system is expressed in matrix form as

$$
\mathbf{g}(\mathbf{u})=\left\{g_{1}(\mathbf{u}), g_{2}(\mathbf{u}), \ldots, g_{m}(\mathbf{u})\right\}^{T}=\mathbf{0} \text { or } g_{k}(\mathbf{u})=0, \mathrm{k}=1 . . \mathrm{m}
$$

By minimization of the augmented potential energy function by incorporating the nonlinear multi freedom constraints, the governing system of equations can be developed (1) as

$$
\min \left\{\Pi(\mathbf{u}): \mathbf{g}(\mathbf{u})=\mathbf{0}, \quad \mathbf{u} \in R^{n}\right\}
$$

The potential energy of the unconstrained finite element model is defined as follows

$$
\Pi(\mathbf{u})=\mathrm{U}(\mathbf{u})-\mathbf{u}^{T} \mathbf{P}=\frac{1}{2} \mathbf{u}^{T} \mathbf{K u}-\mathbf{u}^{T} \mathbf{P}
$$

Where:

- $U(\mathbf{u})$ and $\left(-\mathbf{u}^{T} \mathbf{P}\right)$ : strain energy and external work of system;

- $\mathbf{P}=\left\{\mathrm{P}_{1}, \mathrm{P}_{2}, \ldots, \mathrm{P}_{n}\right\}^{T}:$ global nodal force vector;

- $\mathbf{K}$ : stiffness matrix of global system.

To impose the constraint, adjoin additional unknowns - Lagrange multipliers, form the Lagrangian function as follows

$$
L(\mathbf{u}, \boldsymbol{\lambda})=\left(\frac{1}{2} \mathbf{u}^{T} \mathbf{K u}-\mathbf{u}^{T} \mathbf{P}\right)+\mathbf{g}(\mathbf{u})^{T} \boldsymbol{\lambda}=\left(\frac{1}{2} \mathbf{u}^{T} \mathbf{K} \mathbf{u}-\mathbf{u}^{T} \mathbf{P}\right)+\sum_{k=1}^{m} \lambda_{k} g_{k}(\mathbf{u})
$$

Where: $\lambda=\left\{\lambda_{1}, \lambda_{2}, \ldots, \lambda_{m}\right\}^{T}, \lambda \in R^{m}$ is Lagrange multipliers collected in vector. 
The augmented Lagrange method [8] combines both Lagrange multiplier and penalty function method. The augmented Lagrange function is given by fixing the penalty parameter $\mathrm{w}$ at the start of the iteration as follows

$$
\begin{aligned}
L_{A}(\mathbf{u}, \boldsymbol{\lambda}, \mathbf{w}) & =L(\mathbf{u}, \boldsymbol{\lambda})+\frac{1}{2} \mathrm{w} \mathbf{g}(\mathbf{u})^{T} \mathbf{g}(\mathbf{u})=\left(\frac{1}{2} \mathbf{u}^{T} \mathbf{K u}-\mathbf{u}^{T} \mathbf{P}\right)+\mathbf{g}(\mathbf{u})^{T} \boldsymbol{\lambda}+\frac{1}{2} \mathrm{w} \mathbf{g}(\mathbf{u})^{T} \mathbf{g}(\mathbf{u}) \\
& =\left(\frac{1}{2} \mathbf{u}^{T} \mathbf{K u}-\mathbf{u}^{T} \mathbf{P}\right)+\sum_{k=1}^{m} \lambda_{k} g_{k}(\mathbf{u})+\frac{1}{2} \mathrm{w} \sum_{k=1}^{m} g_{k}^{2}(\mathbf{u})
\end{aligned}
$$

By minimization of the augmented Lagrange multiplier function $L_{A}(\mathbf{u}, \boldsymbol{\lambda}, \mathbf{w})$, a constrained problem is converted into an unconstrained problem, which is expressed in equation (7.1). The Lagrange multipliers are updated in each iteration " $\mathrm{j}$ " using the expressions (7.2) for finding the series of solution $\left\{\boldsymbol{\lambda}_{j}\right\} ;\left\{\mathbf{u}_{j}\right\}$ converged to the optimal solution $\left(\mathbf{u}^{*}, \lambda^{*}\right)$

$$
\left\{\begin{array}{l}
\nabla_{\mathbf{u}} L_{A}(\mathbf{u}, \boldsymbol{\lambda}, \mathbf{w})=\mathbf{K} \mathbf{u}-\mathbf{P}+\nabla_{\mathbf{u}} \mathbf{g}(\mathbf{u})[\boldsymbol{\lambda}+w \mathbf{g}(\mathbf{u})]=\mathbf{0} \\
\boldsymbol{\lambda}_{j+1}=\boldsymbol{\lambda}_{j}+w \mathbf{g}\left(\mathbf{u}_{j}\right)
\end{array}\right.
$$

Equation (7) can be written in compact form as follows

$$
\left\{\begin{array}{l}
\mathbf{K u}+\nabla_{\mathbf{u}} \mathbf{g}(\mathbf{u})[\boldsymbol{\lambda}+w \mathbf{g}(\mathbf{u})]=\mathbf{P} \\
\boldsymbol{\lambda}_{j+1}=\boldsymbol{\lambda}_{j}+w \mathbf{g}\left(\mathbf{u}_{j}\right)
\end{array}\right.
$$

The system of equations $(8.1)$ is nonlinear from constraints $\left(g_{k}(\mathbf{u})=0, \mathrm{k}=1 . . \mathrm{m}\right)$ is nonlinear.

\subsection{Incremental equation}

Method for solving the nonlinear system of equation (8) based on dividing the total load into incremental load step [9,10]. For constructing incremental equation (9.1), utilizing Taylor series formula for a short $\delta \mathbf{u}, \delta \boldsymbol{\lambda}$ to expand function of Eq. (8) around of variable point, keeping only linear term in $\mathbf{u}$, getting

$$
\left\{\begin{array}{l}
\left\{\mathbf{K}+\nabla_{\mathbf{u}}^{2} \mathbf{g}(\mathbf{u}) \boldsymbol{\lambda}+w \nabla_{\mathbf{u}} \mathbf{g}(\mathbf{u}) \nabla_{\mathbf{u}} \mathbf{g}(\mathbf{u})^{T}\right\} \delta \mathbf{u}=\mathbf{P}+\Delta \mathbf{P}-\left\{\mathbf{K u}+\nabla_{\mathbf{u}} \mathbf{g}(\mathbf{u})[\boldsymbol{\lambda}+w \mathbf{g}(\mathbf{u})]\right\} \\
\boldsymbol{\lambda}_{j+1}=\boldsymbol{\lambda}_{j}+w \mathbf{g}\left(\mathbf{u}_{j}\right)
\end{array}\right.
$$

The equation (9) can be expressed in compact form as

$$
\left\{\begin{array}{l}
\mathbf{K}_{\lambda}(\mathbf{u}) \delta \mathbf{u}=\mathbf{P}+\Delta \mathbf{P}-\mathbf{q}_{\lambda}(\mathbf{u}) \\
\boldsymbol{\lambda}_{j+1}=\boldsymbol{\lambda}_{j}+w \mathbf{g}\left(\mathbf{u}_{j}\right)
\end{array}\right.
$$

Where:

$$
\left\{\begin{array}{l}
\mathbf{K}_{\lambda}(\mathbf{u})=\left\{\mathbf{K}+\nabla_{\mathbf{u}}^{2} \mathbf{g}(\mathbf{u}) \lambda+w \cdot \nabla_{\mathbf{u}} \mathbf{g}(\mathbf{u}) \nabla_{\mathbf{u}} \mathbf{g}(\mathbf{u})^{T}\right\} ; \nabla_{\mathbf{u}}^{2} \mathbf{g}(\mathbf{u}) \lambda=\sum_{k=1}^{m} \nabla_{\mathbf{u}}^{2} \mathbf{g}_{k}(\mathbf{u}) \lambda_{k} \\
\mathbf{q}_{\lambda}(\mathbf{u})=\left\{\mathbf{K} \mathbf{u}+\nabla_{\mathbf{u}} \mathbf{g}(\mathbf{u})[\lambda+w \cdot \mathbf{g}(\mathbf{u})]\right\}
\end{array}\right.
$$




\section{Algorithm for solving incremental equations of equilibrium based on Newton Raphson method}

This research establishes the incremental-iterative algorithm for solving the nonlinear modified system of equations based on the Newton Raphson method [11]. In the established incremental formulation the variables including displacement $\mathbf{u}$ and Lagrange multiplier $\lambda$ are updated incrementally corresponding to successive load steps in order to trace out the complete solution path (equilibrium path). The block diagram of algorithm for solving the nonlinear modified system of equations is shown in Fig.2.

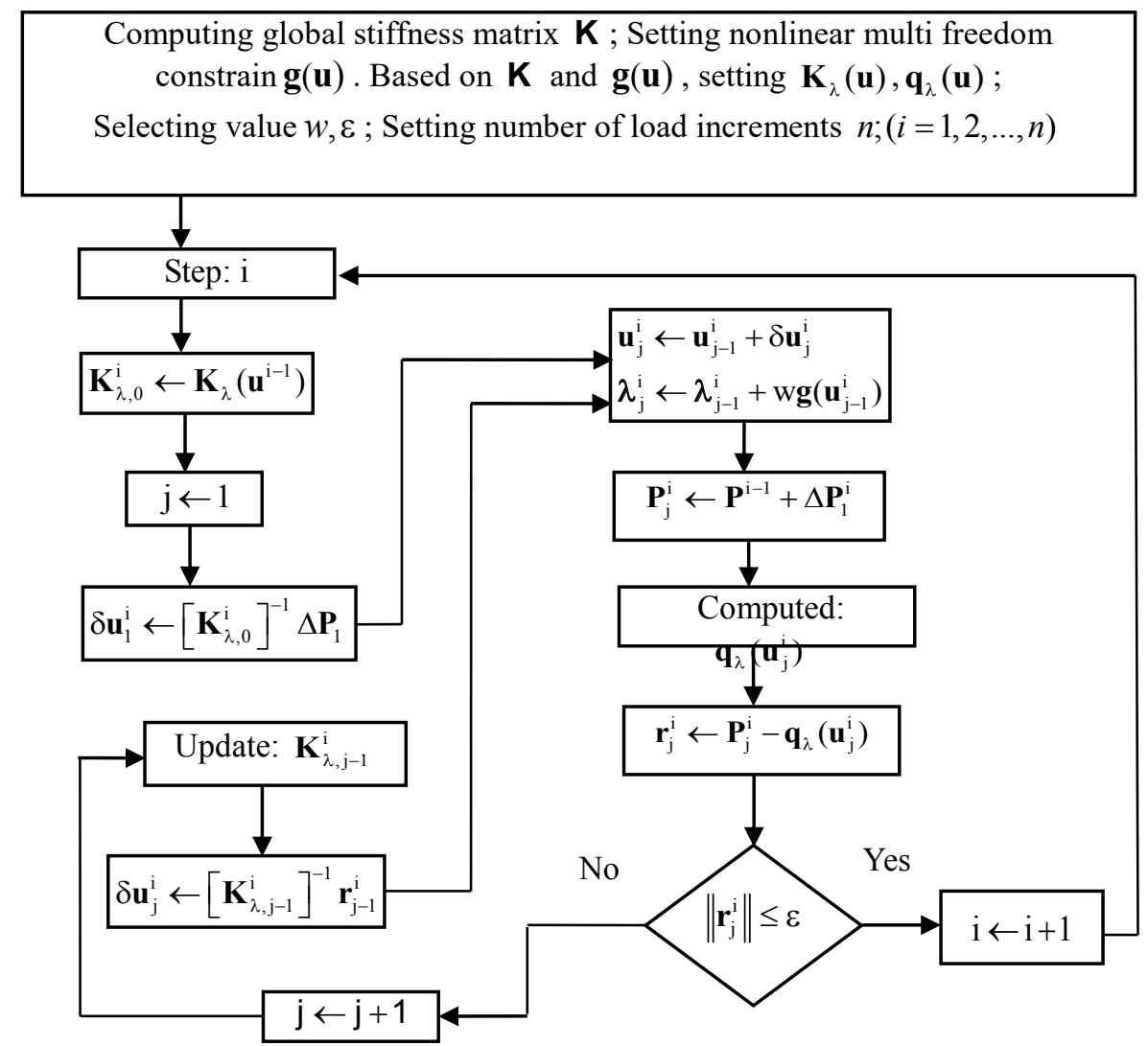

Fig. 1. Incremental-iterative procedure for solving nonlinear modified system of equations based on Newton Raphson technique

\section{Test example}

Based on proposed above incremental-iterative algorithm for solving the nonlinear modified system, the calculation program for static analysis of finite element truss is written in MathCAD software 


\subsection{Example formulation}

The frame is composed of three bars made of the same material and had the same geometrical properties; having nonlinear multi freedom constraints (frame is shown in fig 2). The geometric parameters, material parameters and loading parameters are given

$$
E=2.10^{4}\left(\mathrm{kN} / \mathrm{cm}^{2}\right), A=60 \mathrm{~cm}^{2}, L=420 \mathrm{~cm}, I_{x}=3140 \mathrm{~cm}^{4}, \beta=0.05 .
$$

The nonlinear multi freedom constraints can be is expressed as following functions

$$
\left\{\begin{array}{l}
g_{1}(\mathbf{u})=\left(L+u_{5}-u_{2}\right)^{2}+\left(u_{4}-u_{1}\right)^{2}-L^{2}=0, g_{2}(\mathbf{u})=\left(L+u_{7}-u_{4}\right)^{2}+\left(u_{8}-u_{5}\right)^{2}-L^{2}=0 \\
g_{3}(\mathbf{u})=\left(L+u_{7}-u_{1}\right)^{2}+\left(L+u_{8}-u_{2}\right)^{2}-2 L^{2}=0, g_{4}(\mathbf{u})=u_{1}-\beta u_{2}^{2}=0 \\
\mathbf{g}(\mathbf{u})=\left\{g_{1}(\mathbf{u}), g_{2}(\mathbf{u}), g_{3}(\mathbf{u}), g_{4}(\mathbf{u})\right\}^{T}=\mathbf{0}
\end{array}\right.
$$

For investigating the effectiveness of the proposed algorithm, the research implements the solution process in two ways, including:

- Using the proposed in this paper algorithm established based on the augmented Lagrangian method;

- Using the algorithm established based on penalty function method introduced in [6] by authored.

The problem was solved with different options of weight values $\mathrm{W}$, as following $\mathrm{w} 1=10^{4} ; \mathrm{w} 2=10^{6} ; \mathrm{w} 3=10^{8} ; \mathrm{w} 4=10^{9}$

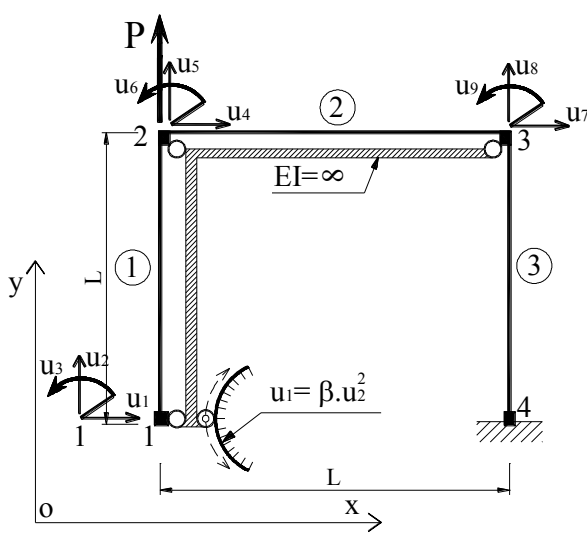

a)

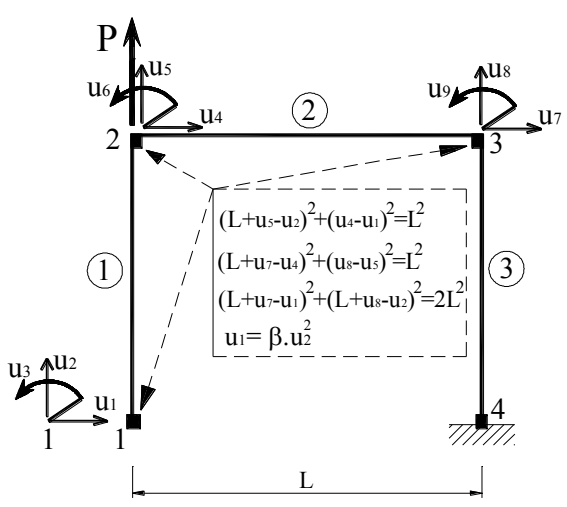

b)

Fig. 2. Examined frame system

\subsection{Numerical results}

The calculation results are load-displacement equilibrium path (shown in fig $3 \& 4$ ). In the diagrams designating equilibrium path as follows: $P_{p}$ - in case using the penalty method; $P_{L}$ - in case using the augmented Lagrangian method 


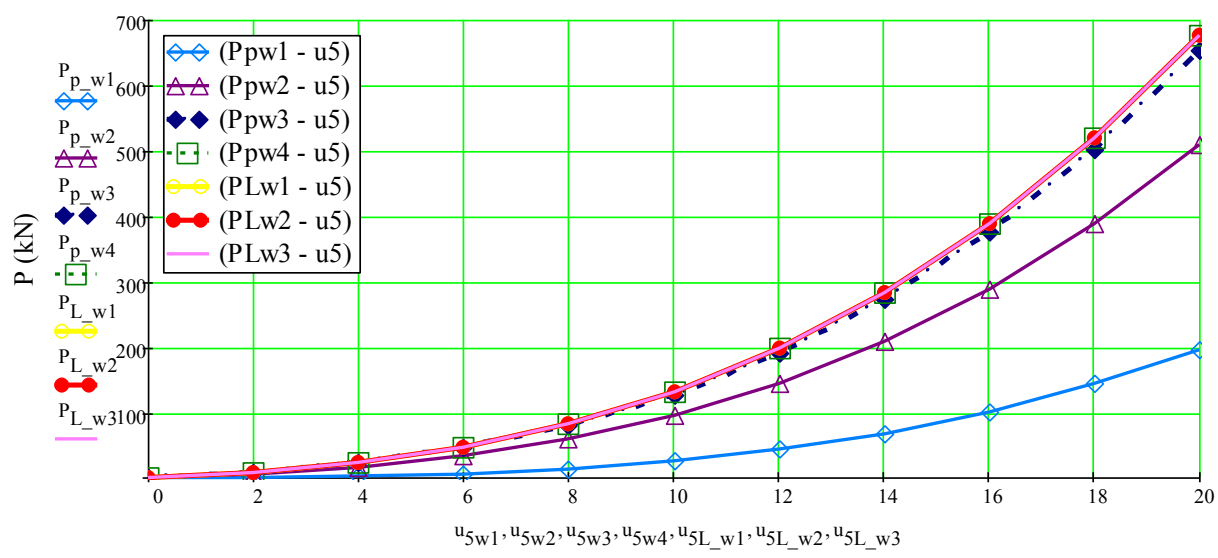

$\mathrm{u} 5(\mathrm{~cm})$

Fig. 3. Load-displacement equilibrium path $\mathrm{P}-\mathrm{u}_{5}$ in cases with different option of weight values

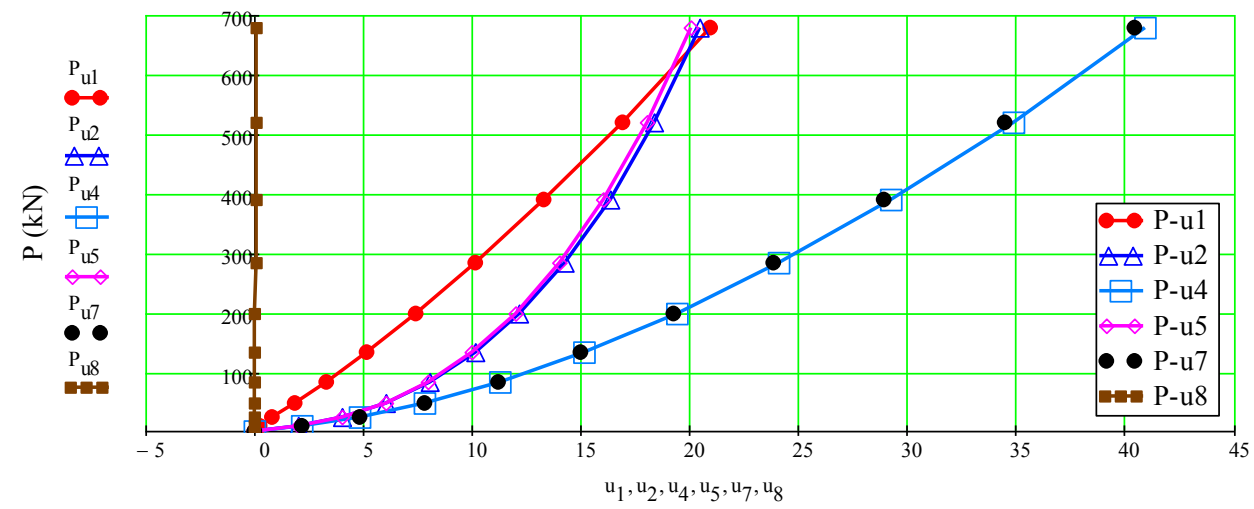

$\mathrm{u}(\mathrm{cm})$

Fig. 4. Load-displacement equilibrium path $\mathrm{P}-\mathrm{u}_{4}$ in cases with different option of weight values

\section{Conclusions}

The numerical results of the investigated frame show that the augmented Lagrangian method is overcome the penalty function method in giving the exact results not depending on the way to choose appropriate weights. Comparing with the Lagrange multiplies method this method has a significant advantage in providing an optimal value of multipliers in addition to the constraints problem solution and generating a quick solution.

\section{References}

1. K.J. Bathe, Finite Element Procedure, (Prentice Hall 2014)

2. O. C. Zienkiewicz, The Finite Element Method for Solid and Structural Mechanics, (Elsevier 2014)

3. J. J. Strodiot, Numerical Methods in Optimization, (Namur - Belgium 2002).

4. V.T.B. Quyen,, D.N. Tien, N.N. Dung, and C.Q. Khanh, IOP Conf. Series: Materials Science and Engineering, 869 (2020) 
5. D. P. Bertsekas, Constrained optimization and Lagrange multiplier method, Athena Scientific, (Academic Press Inc. 1982)

6. V.T.B. Quyen, D.N. Tien, and N.T.L. Huong, Treatment of nonlinear multi freedom boundary constraints in finite element analysis of frame system using Lagrange multiplier method, IOP Conf. Series: Materials Science and Engineering, 960 (2020).

7. K. A. Rajesh, Optimization: Algorithm and Application, (Taylor \& Francis Group 2015)

8. E. G. Birgin, J. M. Martínez, Practical Augmented Lagrangian Methods for Constrained Optimization (Red Hat, Inc 2014)

9. M. A. Crisfield, Nonlinear Finite Element Analysis of Solids and Structures, (John Wiley \& Sons Ltd. 200)

10. W. Sun and Y -X. Yuan, Optimization Theory and Methods - Nonlinear Programming, (Springer 2006).

11. E. Riks, ASME J. Appl. Mech., 39 (4), (1972). 